\title{
Complete response to local therapy for advanced hepatocellular carcinoma with lung metastasis: a case report
}

\author{
Daeun Kim', Seiyeon Park', Won Sohn', Hyun Pyo Hong ${ }^{2}$, Byung lk Kim¹ \\ 'Division of Gastroenterology, Department of Internal Medicine, ${ }^{2}$ Department of Radiology, Kangbuk Samsung Hospital, Sungkyunkwan \\ University School of Medicine, Seoul, Korea
}

Received May 28, 2021

Revised Dec. 6, 2021

Accepted Dec. 28, 2021
The concept of oligometastasis is widely accepted for various types of solid tumors; accordingly, better outcomes can be anticipated with aggressive local interventions. The treatment of advanced hepatocellular carcinoma (HCC) with extrahepatic metastasis is systemic therapy. However, treatment responses to systemic therapy are poor. Recently, a small number of metastatic cancers (oligometastasis) have been controlled by local therapy rather than systemic therapy. Our study reports a case of a 66 -year-old male patient with advanced HCC with lung metastasis, which was treated with local therapy. There were less than four metastases in the lungs, which were treated with wedge resection, radiofrequency, and radiation therapy. He repeatedly underwent local therapy for lung oligometastasis and locoregional therapy for intrahepatic HCC rather than systemic therapy; control by local therapy was possible as his liver function was preserved with Child-Turcotte-Pugh class A. (J Liver Cancer 2022;22:51-56)

Keywords: Hepatocellular carcinoma; Oligometastasis; Transarterial chemoembolization; Radiofrequency ablation; Case report

\section{INTRODUCTION}

Hepatocellular carcinoma (HCC) is one of the most common malignancies worldwide with a high mortality rate. ${ }^{1,2}$ Systemic therapy is the standard treatment for advanced HCC with extrahepatic metastasis. ${ }^{3,4}$ However, local treatment for metastatic HCC has recently been challenged if intrahepatic tumors are well controlled and a small tumor burden of extrahepatic metastasis exists (oligometastasis).

\footnotetext{
Corresponding author: Byung Ik Kim

Department of Medicine, Kangbuk Samsung Medical Center, Sungkyunkwan University School of Medicine, 29 Saemunan-ro, Jongno-gu, Seoul 03181, Korea

Tel. +82-2-2001-2055, Fax. +82-2-2001-2117

E-mail: bik.kim@samsung.com
}

Oligometastases are a clinical state of metastatic disease that is limited in the number of metastatic sites and extent of disease, and amenable to metastasis-directed surgical or ablative therapy. ${ }^{5}$ While metastasis-directed approaches are used for palliation, the primary goal of such treatment for patients with oligometastases is to improve survival and the duration of cancer treatment. ${ }^{5}$ Herein, we report a case of a patient who was disease-free for 6 months after local treatment instead of systemic therapy for oligometastatic HCC in the lungs, which occurred while the intrahepatic tumors were well controlled. This study was approved by the Institutional Review Board of Kangbuk Samsung Hospital (IRB No. 202110-033). This case report was described according to the CARE guidelines available from https://www.care-statement. org/. 


\section{CASE REPORT}

A 66-year-old man presented to our hospital with a large hepatic mass that was detected by ultrasonography screening at another hospital. He had been diagnosed with chronic hepatitis B 12 years ago, but had no history of any antiviral treatment. There were no associated symptoms or remarkable findings on physical examination. The results of the initial blood tests were as follows: white blood cell count, $6,400 / \mu \mathrm{L}$; hemoglobin, $15.5 \mathrm{~g} / \mathrm{dL}$; platelet count, $234,000 / \mu \mathrm{L}$; total bili-
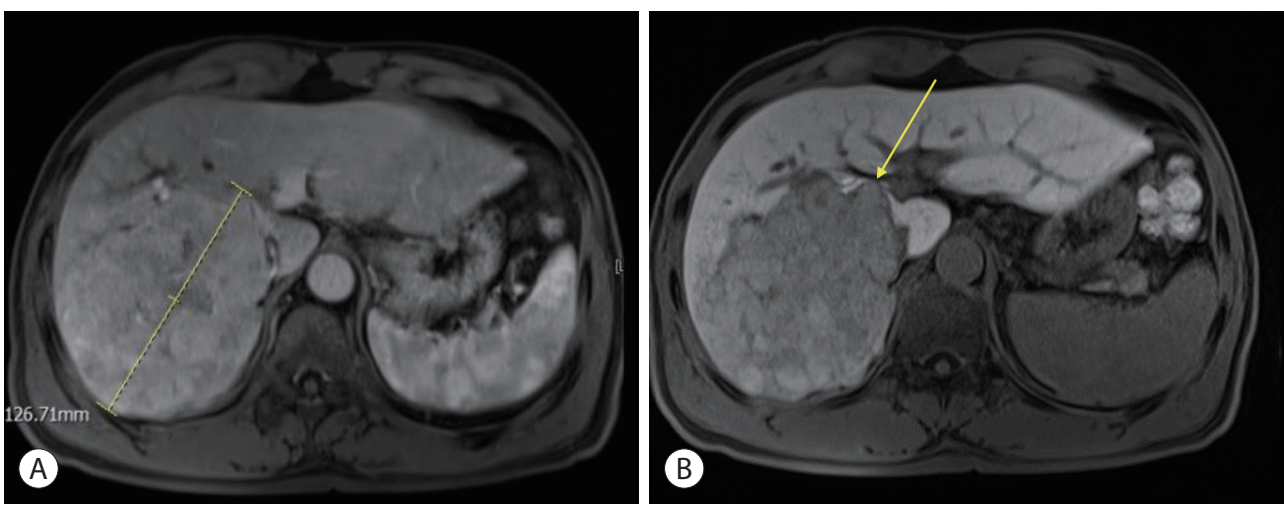

Figure 1. A huge hepatocellular carcinoma $(13 \mathrm{~cm})$ in the right hepatic lobe is shown on initial liver magnetic resonance imaging. (A) T1W arterial phase. (B) Hepatobiliary phase (yellow arrow).
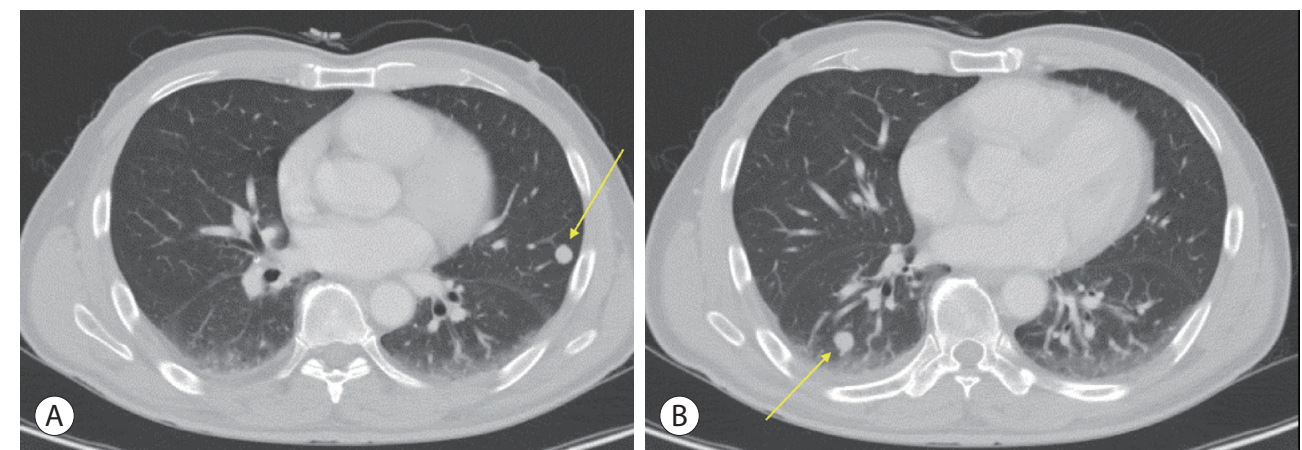

Figure 2. After initial treatment, chest computed tomography shows lung metastases (yellow arrow). (A) Left upper lung nodule. (B) Right lower lung nodule.
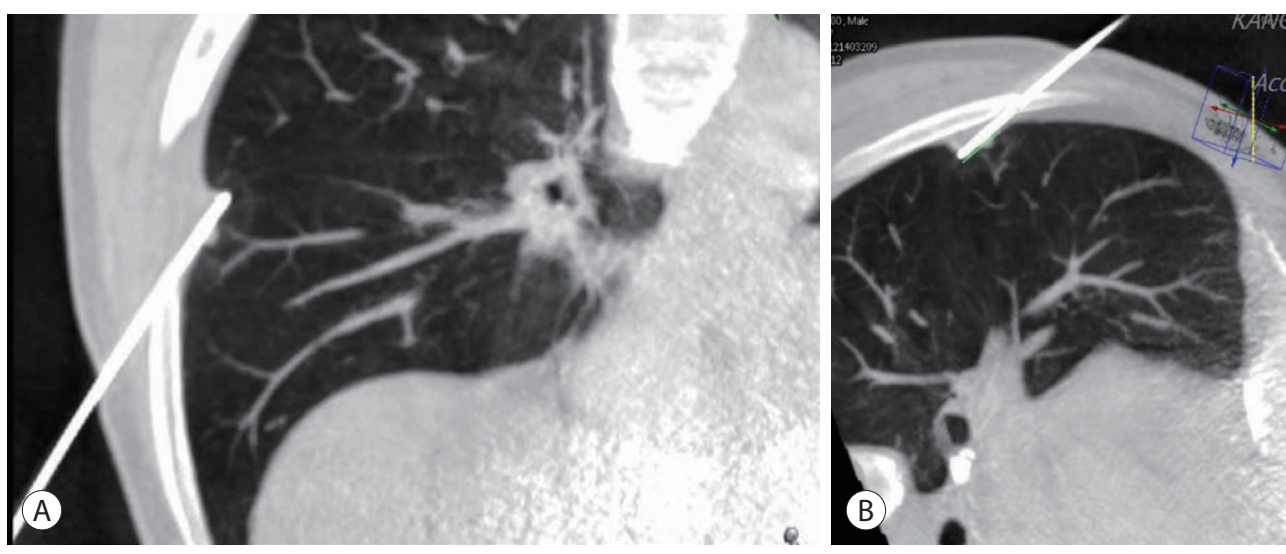

Figure 3. Radiofrequency ablation was performed on the subpleural lung metastasis. (A) Coronal view. (B) Sagittal view. 
rubin, $0.76 \mathrm{mg} / \mathrm{dL}$; serum albumin, $3.8 \mathrm{~g} / \mathrm{dL}$; prothrombin time-international normalized ratio, 1.12; aspartate aminotransferase, $89 \mathrm{IU} / \mathrm{L}$; and alanine aminotransferase, $60 \mathrm{IU} / \mathrm{L}$. The hepatitis B serologic tests were as follows: hepatitis B surface antigen negative, anti-HBs positive, and anti-HBc (hepatitis B core) Ab positive. The level of alpha-fetoprotein (AFP) exceeded 60,500 ng/mL, and the protein induced by the absence of vitamin K or antagonist-II (PIVKA-II) level was $26,890 \mathrm{mAU} / \mathrm{mL}$. Liver function was preserved with Child-Turcotte-Pugh (CTP) class A, and the Eastern Cooperative Oncology Group performance status was 1.

The initial liver magnetic resonance imaging (MRI) demonstrated an approximately $13-\mathrm{cm}$ large HCC in the right hepatic lobe (segment VI) with the typical radiological features, including hypervascularity in the arterial phase and

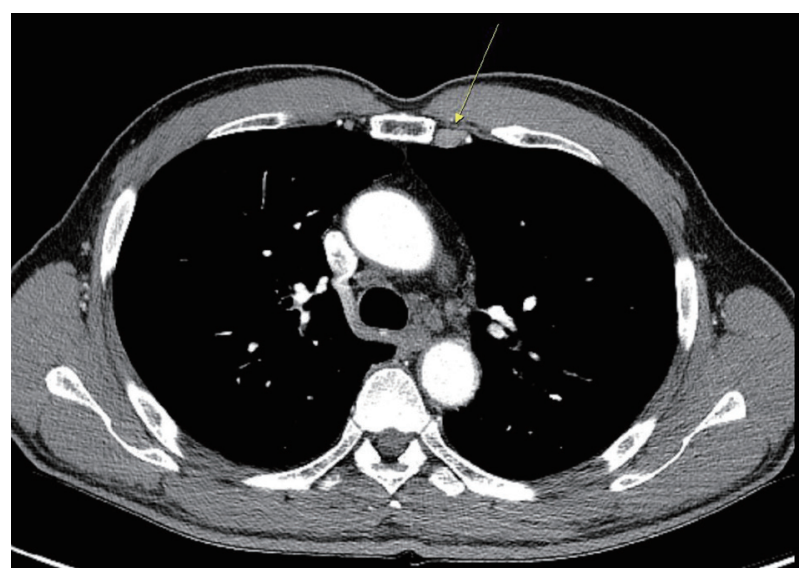

Figure 4. After approximately 6 years of initial treatment, chest computed tomography shows a slightly increased lymph node (arrow) size of $7 \times 15.6 \mathrm{~mm}$ in the left internal mammary chain. washout in the portal and delayed phases. Direct invasion was observed in the right anterior and posterior portal veins (Fig. 1).

The patient was diagnosed with advanced-stage HCC (Barcelona Clinic Liver Cancer stage C; modified Union for International Cancer Control T3N0M0, stage III) based on the typical imaging findings. A liver biopsy was not performed. Transarterial chemoembolization (TACE) was performed to treat the intrahepatic HCC, and radiotherapy was planned for the right anterior and posterior portal vein invasion (4,000 cGy, $400 \mathrm{cGy} \times 10$ sessions).

Liver computed tomography (CT) or MRI was subsequently performed every 3 months, and there was a marginal HCC recurrence in the left lateral segment, which was additionally treated with TACE and radiofrequency ablation (RFA). Within 10 months, the HCC was treated four times with TACE, once with RFA, and once with radiotherapy.

Eleven months after the initial treatment, follow-up liver CT showed a local recurrence in the right lobe (segment VI), and chest CT revealed two nodules in both lungs (right lower lobe and left upper lobe) (Fig. 2). The AFP level increased to $906.4 \mathrm{ng} / \mathrm{mL}$, but the CTP class was A. Since the patient's liver function was preserved with a CTP score of A, local treatment was performed for the intrahepatic lesions and lung metastases: a second RFA was performed for the marginal recurrence in segment VI, and wedge resection was performed using video-assisted thoracic surgery for the pulmonary lesions. Metastatic HCC was pathologically confirmed in the wedge resection specimens.

Fourteen months after the initial treatment, chest CT
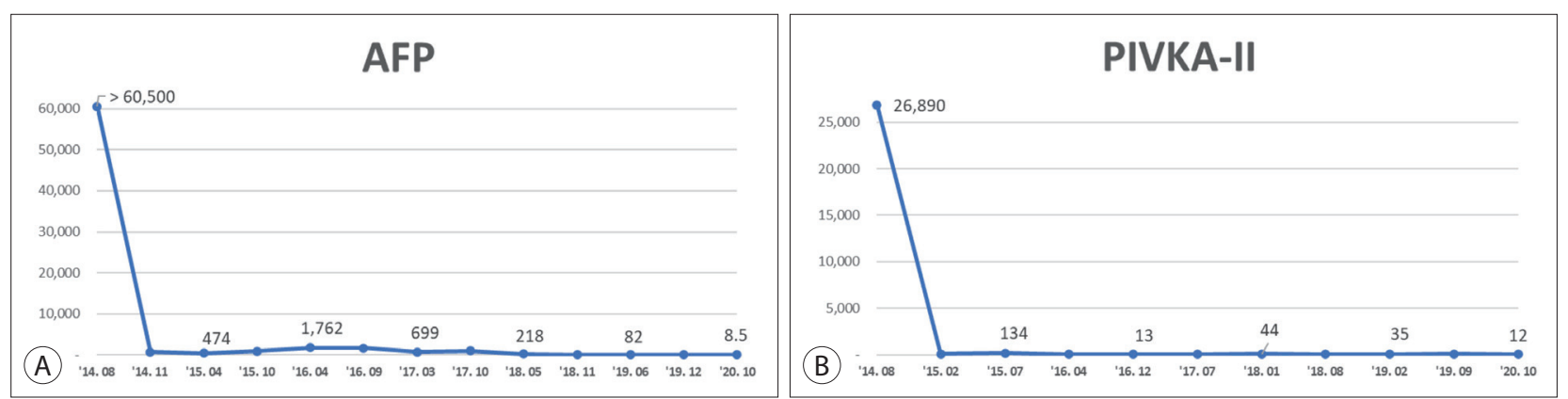

Figure 5. Serum tumor makers significantly decreased after initial treatment and mildly increased at the first relapse. (A) Alpha-fetoprotein (AFP). (B) Protein induced by the absence of vitamin K or antagonist-II (PIVKA-II). 
showed a new metastatic lesion in the right upper lobe of the lung, and RFA was performed for this nodule. Two years later, chest CT detected another new lesion suspicious of metastasis in the right middle lobe; therefore, a second lung RFA was performed (Fig. 3).

Subsequently, imaging tests were performed every 3 months, and a small marginal recurrence was observed in the liver. However, there were no metastatic lesions on chest CT. Intrahepatic HCC was well controlled by additional TACE.

After approximately 6 years of initial treatment, viable HCC was observed in the right lobe (segment VI) of the liver, and no new lesions were detected on chest CT. However, a lymph node with a size of $7 \times 15.5 \mathrm{~mm}$ was identified in the left internal mammary chain (Fig. 4). Metastatic lymph nodes were also detected on positron emission tomography examination. Based on a multidisciplinary approach, it was decided to conduct radiotherapy for the intrahepatic lesion (segment VI) (3,200 cGy, $800 \mathrm{cGy} \times 4$ sessions) and the left internal mammary chain in the lung (4,000 cGy, $400 \mathrm{cGy} \times 10$ sessions).

In summary, oligometastases with recurrent HCCs were repeatedly treated with local therapies (surgical resection, RFA, and radiotherapy), as the intrahepatic tumors were well controlled. After initial treatment, AFP significantly decreased from over $65,000 \mathrm{ng} / \mathrm{mL}$ to $664 \mathrm{ng} / \mathrm{mL}$, and PIVKAII decreased from $26,890 \mathrm{mAU} / \mathrm{mL}$ to $26 \mathrm{mAU} / \mathrm{mL}$ (Fig. 5). No tumor recurrence was observed in the last CT scan. The patient is currently tumor-free for 6 months after the last treatment (Fig. 6).

\section{DISCUSSION}

The overall survival of patients with HCC has been gradually improving due to the development of various treatment methods. ${ }^{6}$ Nonetheless, the prognosis is still poor in patients with advanced HCC with extrahepatic metastasis. ${ }^{7}$ Generally, in the case of extrahepatic metastasis, systemic chemotherapy is the treatment of choice. ${ }^{3}$ However, the survival benefit of systemic chemotherapy in patients with advanced HCC is lacking. ${ }^{2}$ In two randomized clinical trials, the median survival gain in patients with advanced HCC receiving sorafenib was only 2.3-2.8 months compared to that in the placebo group. ${ }^{8}$
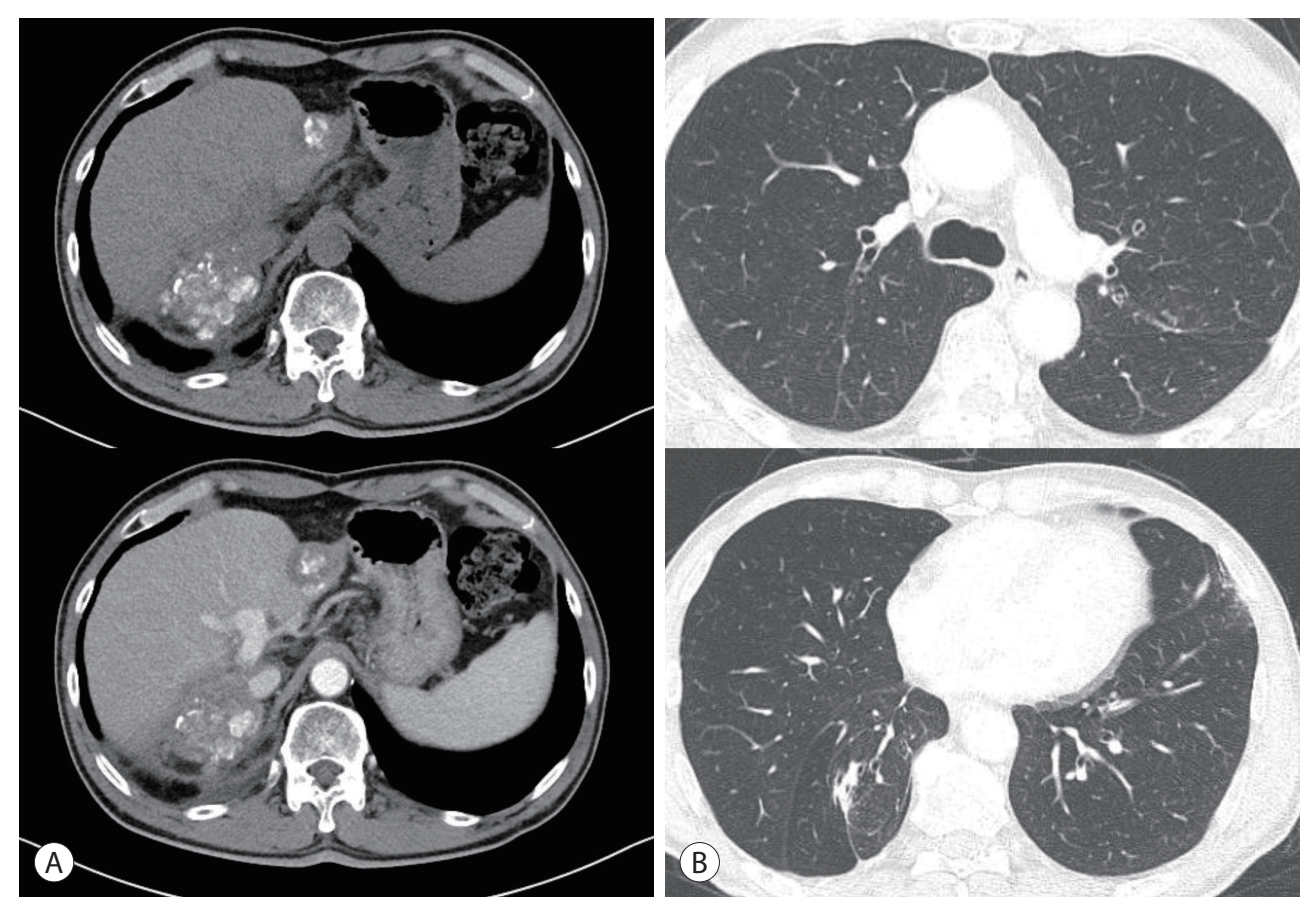

Figure 6. Last computed tomography (CT) shows no evidence of hepatocellular carcinoma recurrence. (A) Liver CT. (B) Chest CT. 
For decades, surgical resection has been recognized as the standard treatment for liver metastases of colorectal cancer if possible. In addition, local consolidative therapy for oligometastases in non-small cell lung cancer was shown to be beneficial in a randomized phase II study. ${ }^{9,10}$

Systemic therapy is the standard treatment for HCC with extrahepatic metastasis. However, systemic therapy in advanced HCC is poorly tolerated owing to adverse effects, resulting in a relatively poor disease control rate. Therefore, local treatment for metastatic HCC has been challenging in some instances. It might be helpful in the case of oligometastatic HCC if the intrahepatic tumor is well controlled. ${ }^{11}$ In addition, the advantage of resection or ablation in oligometastases over systemic therapy is that there are no adverse effects that may occur due to continuous drug administration, which is the cause of poor tolerability in systemic therapy. In addition, the reduction in tumor burden could be confirmed by imaging (CT or MRI) immediately after the treatment.

Previous studies on the local treatment of HCC with oligometastases have been as follows: first, Gerum et al. ${ }^{12}$ reported that stereotactic body radiation therapy resulted in a high local control rate and acceptable survival rates in patients with oligometastatic HCC. Overall survival was predicted by the number of lesions, performance status, and pretreatment liver function in patients with HCC. Second, surgical resection might be beneficial as a treatment modality for pulmonary metastasis of HCC. ${ }^{13}$ Finally, local ablation therapy could be helpful in treating oligometastatic HCC, as in this case. Li et al. ${ }^{14}$ showed that RFA could be a therapeutic option in HCC patients with pulmonary metastasis. Lassandro et al. ${ }^{15}$ reported that RFA was beneficial to patients with pulmonary metastasis of HCC in terms of treatment efficacy and safety.

In this case, liver function was preserved with CTP score A, and intrahepatic lesions were well controlled with TACE and RFA. Therefore, local treatment was possible for oligometastatic HCC in the lung. Thus, in advanced HCC, if intrahepatic HCC is well controlled and oligometastases occur, local therapy may be beneficial, if feasible.

\section{Conflicts of Interest}

The authors have no conflicts of interest to disclose.

\section{Ethics Statement}

This study was approved by the Institutional Review Board (IRB number: 2021-10-33), and the requirement for informed consent was waived owing to the use of preexisting medical records.

\section{Funding Statement}

No funding to declare.

\section{Data Availability}

Data sharing not applicable to this article as no datasets were generated or analyzed for this case report.

\section{ORCID}

$\begin{array}{ll}\text { Daeun Kim } & \text { https://orcid.org/0000-0001-5116-3537 } \\ \text { Seiyeon Park } & \text { https://orcid.org/0000-0003-4312-7303 } \\ \text { Won Sohn } & \text { https://orcid.org/0000-0003-3284-2715 } \\ \text { Hyun Pyo Hong } & \text { https://orcid.org/0000-0002-9864-7739 } \\ \text { Byung Ik Kim } & \text { https://orcid.org/0000-0002-7215-7715 }\end{array}$

\section{Author Contribution}

\author{
Conceptualization: DK, BIK, WS \\ Data curation: DK, BIK, WS \\ Methodology: DK, BIK, WS \\ Project administration: BIK, WS \\ Writing original draft: DK, BIK, WS \\ Writing review \& editing: BIK, WS, SP, HPH \\ Approval of final manuscript: all authors.
}

\section{References}

1. Forner A, Llovet JM, Bruix J. Hepatocellular carcinoma. Lancet 2012;379:1245-1255.

2. Jung SM, Jang JW, You CR, Yoo SH, Kwon JH, Bae SH, et al. Role of intrahepatic tumor control in the prognosis of patients with hepatocellular carcinoma and extrahepatic metastases. J Gastroenterol Hepatol 2012;27:684-689.

3. Heimbach JK, Kulik LM, Finn RS, Sirlin CB, Abecassis MM, Roberts $L R$, et al. AASLD guidelines for the treatment of hepatocellular carcinoma. Hepatology 2018;67:358-380.

4. European Association for the Study of the Liver. EASL clinical practice guidelines: management of hepatocellular carcinoma. J Hepatol 2018;69:182-236. 
5. Milano MT, Biswas T, Simone CB 2nd, Lo SS. Oligometastases: history of a hypothesis. Ann Palliat Med 2021;10:5923-5930.

6. Natsuizaka M, Omura T, Akaike T, Kuwata Y, Yamazaki K, Sato T, et al. Clinical features of hepatocellular carcinoma with extrahepatic metastases. J Gastroenterol Hepatol 2005;20:1781-1787.

7. Ochiai T, Ikoma H, Okamoto K, Kokuba Y, Sonoyama T, Otsuji E. Clinicopathologic features and risk factors for extrahepatic recurrences of hepatocellular carcinoma after curative resection. World J Surg 2012;36:136-143.

8. Korean Liver Cancer Association (KLCA); National Cancer Center (NCC). 2018 Korean Liver Cancer Association-National Cancer Center Korea practice guidelines for the management of hepatocellular carcinoma. Korean J Radiol 2019;20:1042-1113.

9. Timmerman RD, Bizekis CS, Pass HI, Fong Y, Dupuy DE, Dawson $L A$, et al. Local surgical, ablative, and radiation treatment of metastases. CA Cancer J Clin 2009;59:145-170.

10. Gomez DR, Blumenschein GR Jr, Lee JJ, Hernandez M, Ye R, Camidge DR, et al. Local consolidative therapy versus maintenance therapy or observation for patients with oligometastatic nonsmall-cell lung cancer without progression after first-line systemic therapy: a multicentre, randomised, controlled, phase 2 study.
Lancet Oncol 2016;17:1672-1682.

11. Abdel-Rahman 0 . Role of liver-directed local tumor therapy in the management of hepatocellular carcinoma with extrahepatic metastases: a SEER database analysis. Expert Rev Gastroenterol Hepatol 2017;11:183-189.

12. Gerum S, Heinz C, Belka C, Walter F, Paprottka P, De Toni EN, et al. Stereotactic body radiation therapy (SBRT) in patients with hepatocellular carcinoma and oligometastatic liver disease. Radiat Oncol 2018;13:100.

13. Omimaru Y, Sasaki Y, Yamada T, Eguchi H, Takami K, Ohigashi H, et al. The significance of surgical resection for pulmonary metastasis from hepatocellular carcinoma. Am J Surg 2006;192:46-51.

14. Li X, Wang J, Li W, Huang Z, Fan W, Chen Y, et al. Percutaneous CT-guided radiofrequency ablation for unresectable hepatocellular carcinoma pulmonary metastases. Int J Hyperthermia 2012;28:721728.

15. Lassandro G, Picchi SG, Bianco A, Di Costanzo G, Coppola A, lerardi $A M$, et al. Effectiveness and safety in radiofrequency ablation of pulmonary metastases from HCC: a five years study. Med Oncol 2020;37:25. 